\title{
A Critical Review of PAHs as DIB Carriers - Progress and Open Questions
}

\author{
F. Salama ${ }^{1}$ and P. Ehrenfreund ${ }^{2}$ \\ ${ }^{1}$ NASA Ames Research Center, Space Science \& Astrobiology Division, \\ Moffett Field, California 94035, USA \\ email: farid.salama@nasa.gov \\ ${ }^{2}$ Space Policy Institute, George Washington University Washington DC, USA \\ email: pehren@gwu.edu
}

\begin{abstract}
PAHs are among the most commonly proposed and popular candidates for DIB carriers. We present a critical assessment of the PAH-DIB model in view of the progress and the advances that have recently been achieved through a series of complementary studies involving astronomical observations of DIBs, laboratory simulation of interstellar analogs for PAHs (neutrals and ions), space exposure experiments of PAHs, theoretical calculations of PAH spectra and the modeling of diffuse and translucent interstellar clouds. What have we learned from these complementary studies? What are the constraints that can now be derived for the PAHs as DIB carriers? What are the strengths and the weaknesses of the PAH model to account for the DIBs?
\end{abstract}

Keywords. ISM: lines and bands, ISM: molecules, ions, laboratory, molecular data

\section{Introduction}

DIB carriers are a ubiquitous presence in the current and early Universe and are clearly correlated to the amount of dust and the prevailing UV field. There is a broad consensus in the science community around the notions that DIB carriers are carbon indicators, chemically stable and that they exhibit, in some cases, substructure indicative of rotational contours of complex gas phase molecules. Among all the carbon materials thought to be present in space (Henning \& Salama, 1998), PAHs are a class of very stable aromatic organic molecules made only of fused carbon benzenoid rings and hydrogen atoms. PAH molecules, in their neutral and ionized forms, play an important role in the interstellar medium due to the rich chemistry of carbon and its ability to form a large variety of complex molecular species involving hydrogen - the most abundant element (for a recent review see Salama 2008). The PAH-DIB proposal has been put forward on the basis of the expected abundance of PAHs in the interstellar medium, their physical and chemical properties including their characteristic spectral signatures and their stability against UV photodissociation (Van der Zwet \& Allamandola 1985; Léger \& d'Hendecourt 1985; Crawford et al. 1985; Salama et al. 1996). PAHs are now thought to be largely responsible for the discrete infrared emission bands observed at 3.3, 6.2, 7.7, 8.6 and $11.3 \mu \mathrm{m}$ in many astronomical objects including HiI regions, planetary and reflection nebulae, and the ISM of the Milky Way and other galaxies (Tielens 2008). Observations from the IR space satellites ISO (Mattila et al. 1996), IRTS (Onaka et al. 1996) and Spitzer (Flagey et al. 2006) have confirmed that PAHs are ubiquitous throughout the general diffuse ISM as well. According to the astrophysical model, PAHs are expected to be present as a mixture of free, neutral and ionized, molecules following a broad size distribution which ranges from small, gas-phase, molecules ( $\leqslant 25$ carbon atoms) to large graphitic platelets (Allamandola et al. 1989; Puget \& Léger, 1989). PAHs form a link 
between the gas and the solid phase of interstellar dust and are a key element for the coupling of stellar FUV photons with the interstellar gas.

Extensive laboratory studies have been performed to measure the UV and visible spectra of neutral and ionized PAHs in media that are astrophysically relevant, i.e., media where the neutral PAH molecules and their ions are free and isolated at low temperature. First, neutral PAH molecules and ions were isolated at $4.2 \mathrm{~K}$ in solid neon matrices (see Salama 1999 and references therein). Based on these preliminary data and on the astronomical data then available, the PAH-DIB proposal was re-assessed (Salama et al. 1996) and the laboratory data assessed against extensive surveys of reddened early-type stars (Salama et al. 1999; Ruiterkamp et al. 2002; Ruiterkamp et al. 2005). The results of these first surveys led to the unescapable conclusion that although PAHs (neutral and ions) were promising candidates for the DIB carriers, laboratory gas-phase data were imperatively needed for a quantitative comparison with astronomical data.

The evolution witnessed over the past decade in the size and in the quality of the astronomical and the laboratory data sets is the result of major advances in detector technology and in laboratory techniques. As a result, an extensive set of gas-phase spectra of neutral and ionized PAHs is now available (for a recent review see Salama 2008 and references therein) making it possible for the first time to unambiguously search for the signature of specific PAHs in the diffuse interstellar medium (Salama et al. 2011; Gredel et al. 2011) and to quantitatively test the PAHs as potential DIB carriers. These are probably the major advances that can be noted on the 'PAH-DIB' front since the last DIB conference held in 1994 (Tielens and Snow 1995).

As a direct result of these advances and the concomitant advances achieved through complementary studies involving astronomical observations of DIBs, laboratory simulation of interstellar analogs for neutral and ionized PAHs (Salama 2008 and references therein; Salama et al. 2011; Gredel et al. 2011), space exposure experiments of PAHs (Ehrenfreund et al. 2007; Bryson et al. 2011; Mattioda et al. 2012), theoretical calculations of PAH spectra (Weisman et al. 2003; Ruiterkamp et al. 2005) and the modeling of diffuse and translucent interstellar clouds (Snow and McCall 2006 and references therein) it has become possible to further test the validity of the 'PAH-DIB' proposal.

Here, we present a critical assessment of the PAH-DIB model in view of these progresses: what have we learned from these complementary studies? what are the constraints that can now be derived for the PAHs as DIB carriers? what are the strengths and the weaknesses of the PAH model to account for the DIBs?

\section{Comparison of DIB observations with PAH data}

A few major conclusions can be derived from the comparative survey of laboratory and astronomical data performed in the context of the PAH-DIB proposal.

* A global examination of the spectra of the PAH molecules measured in cold free jet expansions confirms the general PAH spectral characteristics that were previously derived and reported for PAHs measured in solid inert gas matrices (Salama et al. 1999), i.e., that PAH spectra can generally be characterized by $1-2$ strong features and several related weak bands. The weaker bands associated with a specific PAH are expected to be observed only when the strongest PAH feature is associated with one of the strong or moderately strong, well-known, DIBs. Inversely, if the strongest feature of a PAH corresponds to one of the weak DIBs observed in the astronomical spectra there is, obviously, no possibility of detecting the weaker PAH band relatives which are estimated to be not deeper than 0.001 of the continuum. In other words, there are two scenarios which can be met while attempting to identify specific DIBs with specific PAHs: (i) a situation where many 
absorption bands of a single PAH correlate with a combination of strong and weak DIBs. This is the most rewarding situation where a decisive and unambiguous spectral identification can be made based on the comparison of the wavelength positions, energy intervals and relative intensities of numerous bands, (ii) a situation where the strongest absorption band of a PAH correlates with a weak DIB. This in itself does not represent a spectral fit. The assignment gains credibility, however, if a significant number of PAHs are found to be correlated with weak DIBs along many lines of sight and the correlation is found to increase with reddening.

* The spectra of the PAH molecules and ions measured in cold free jet expansions allow to measure the intrinsic band profiles and absolute band peak positions and a direct comparison with the astronomical observations. Close examination of the spectra indicates that the bands associated with the ionic transitions are generally broader than the bands associated with the neutral molecules. The vibronic bands of neutral PAHs measured so far in the laboratory typically range between 2 and $10 \mathrm{~cm}^{-1}$ while the vibronic bands associated with the ions typically range between 25 and $145 \mathrm{~cm}^{-1}$ (Salama 2008 and references therein).

* Most importantly, recent astronomical surveys (Salama et al. 2011; Gredel et al. 2011) have shown that the direct comparison of the spectra of a limited sample of neutral PAH molecules measured under relevant astrophysical laboratory conditions (i.e., free, cold molecules) with astronomical spectra at a high signal-to-noise ratio measured along the lines of sight of a set of selected reddened stars does not lead to the identification of DIBs. In all cases, the stellar spectra do not contain telluric features which could prevent the detection of weak PAH bands. Accurate upper limits for the abundances of the neutral PAHs population along those lines of sight were derived from these comparisons. Typical fractional abundances ranging between $10^{-10}$ and $10^{-8}$ for the specific PAH molecules probed along specific lines of sight. Similar surveys still need to be achieved to search for the signature of ionized PAHs. This task is however more challenging because the bands associated with PAH ions are broad and more difficult to extract from the baseline of the observations.

* The gas-phase laboratory spectra of jet-cooled PAHs show that neutral PAHs exhibit intrinsic band profiles that are closely similar to the profiles of some DIBs. The case is strikingly illustrated in the comparison of the $5362.8 \AA$ band of neutral pentacene, $\left(\mathrm{C}_{22} \mathrm{H}_{14}\right)$, with the narrow $2 \AA$ full width at half maximum (FWHM) 5363.8 $\AA$ DIB detected along the individual lines-of-sight we observed in our survey (Fig. 1). The laboratory band for pentacene exhibits a $1.9 \AA$ FWHM while the DIB feature extracted from the average spectrum shows a $2.1 \AA$ FWHM. However, because the laboratory spectrum of $\mathrm{C}_{22} \mathrm{H}_{14}$ exhibits another band of comparable strength at $5339.5 \AA$ where only a weaker feature is found in the astronomical spectra the pentacene molecule is ruled out as a potential band carrier for the $5363.8 \AA$ DIB. Similar observations can be derived from the comparison of the absorption bands of pyrene $\mathrm{C}_{16} \mathrm{H}_{10}$ with the set of weak DIBs observed in the 3160-3170 A range. Here too, both sets of bands have similar FWHM values (of the order of $1.0 \AA$ ). Here too, again, the non-observation of DIBs at the position of of the stronger pyrene absorption bands in the $3200-3210 \AA$ range rules out the pyrene molecule as a potential band carrier for the DIBs seen in the $3160-3170 \AA$ region.

This shows, however, that it is now possible to unambiguously search for the signature of specific PAH molecules and ions in the lines-of-sight of diffuse and translucent clouds through a close comparison between intrinsic band peak positions and band structure (band profiles). This information cannot be obtained from IR data alone that is not characteristic enough and can only be retrieved from the UV and optical ranges. This is a requisite for demonstrating the presence (or the absence) of PAHs in the diffuse ISM. 

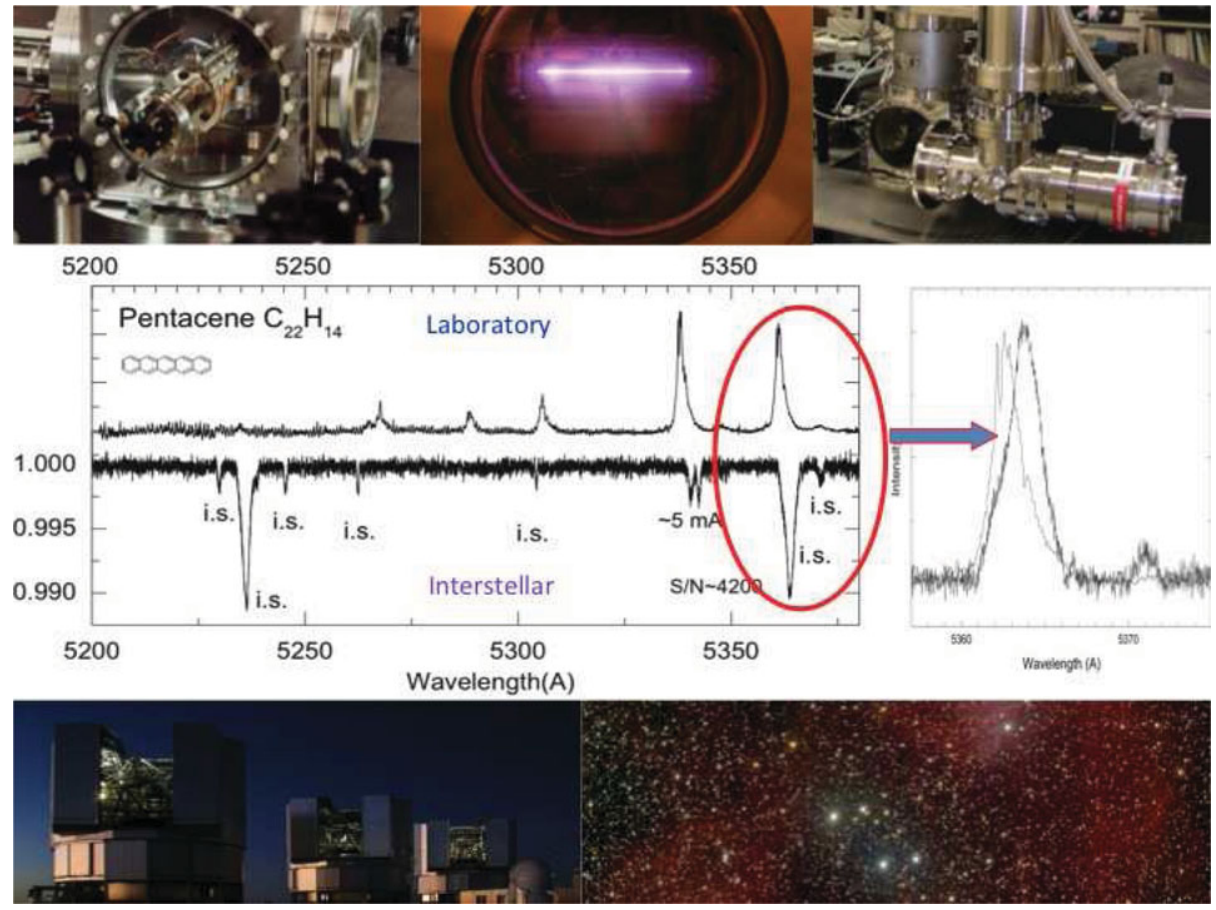

Figure 1. Comparison of the intrinsic bandwidths and band peak positions of vibronic bands in the laboratory absorption spectrum of jet-cooled PAH pentacene $\left(\mathrm{C}_{22} \mathrm{H}_{14}\right)$ with the band characteristics of the diffuse interstellar bands in the average spectrum measured toward a set of selected translucent clouds. (Adapted from Salama et al. 2011)

A new era opens that will hopefully allow to detect and/or rule-out specific PAHs in space and fully test the PAH proposal.

More astronomical surveys of DIB profiles toward an extensive set of reddened stars in combination with improved modeling of stellar lines are now needed, especially in the ranges which have not been as yet frequently observed (NUV and NIR). More laboratory measurements of direct absorption spectroscopy are also needed to measure the spectra of larger PAH molecules and their ions (both positive and negative) as well as radicals (hydrogenated and dehydrogenated PAHs) and substituted PAHs (see e.g., Rouille et al. this volume). A new era begins for the search of complex molecules in space.

\section{Implications}

A critical review of PAHs as DIB carriers has shown that major progress has been achieved in our understanding of the spectral properties of neutral and ionized PAHs since the last meeting devoted to the DIBs in 1994. We can now use laboratory data that can be directly compared to the astronomical observations. The laboratory database is still limited in size though and it only surveys the "tip of the iceberg" for this class of chemicals. Many additional space parameters that involve PAHs of greater molecular sizes, of different structures and composition (e.g., hydrogenated, de-hydrogenated, involving hetero atoms. etc...) still need to be explored. Quantum theoretical calculation techniques have also largely evolved in accuracy and in resolution over the past decade allowing for an increased guiding role for theory in the upcoming years. The ultimate 
answer will still have to come, however, from laboratory measurements performed under relevant conditions for proper comparison with diffuse interstellar medium data.

Open questions remain:

* Have PAHs been detected in the UV-Visible? The answer is "No", no firm identification of DIBs with PAHs (ions or neutrals) have been found so far. The lack of the detection of spectral signature(s) in the UV-Visible range that can be attributed to (or related to) PAHs still represents a challenge to the general PAH model. Although the lack of signature in the UV range could be explained by the overlap of multiple broad bands, the lack of visible signatures is puzzling.

* Can/Should PAHs be ruled out as DIB carriers? No, or at least, not yet. Although it is tempting to rule out a potential carrier for the DIBs, the problem of band widths (PAH ions bands are broad and difficult to extract unambiguously from the observations) pointed out above combined with the fact that PAHs still fulfill the major requirements for band carries in the diffuse ISM (elemental abundances, stability, variety) shows that one needs be careful before ruling out PAH as DIB carriers.

* What is the future of the PAH-DIB Proposal? It is clear from the discussion above that astrophysical models that take advantage of the new knowledge gained regarding the basic properties of molecular PAHs (neutrals and ions) are needed. This modeling, together with "3rd generation" laboratory experiments that will further push the size limits for PAH molecules and ions that can be measured cold and isolated, and advanced, more elaborate, theoretical calculations should help progress in this field.

* Looking beyond the DIB issue: We have to keep in mind that PAHs are part of the "carbon universe" and, as such, they should be detected (confirmed) at other wavelengths (UV, Visible or FIR). This is probably the most important question that is now facing the $\mathrm{PAH}$ model.

\section{Acknowledgements}

Support from NASA SMD through the APRA Laboratory Astrophysics Program (F.S.) and the NAI (P.E.) is gratefully acknowledged .

\section{References}

Allamandola L. J., Tielens A. G. G. M., \& Barker, J. R. 1989, ApJS, 71, 733

Bryson, K. L., Peeters Z., Salama F., et al. 2011, Advances in Space Research, 48, 1980

Crawford, M. K., Tielens, A. G. G. M., \& Allamandola, L. J. 1985, ApJ, 293, L45

Ehrenfreund, P., Ruiterkamp R., Peeters Z., et al. 2007, Planetary and Space Science, 55, 383

Flagey, N., Boulanger, F., Verstraete, L., et al. 2006, A\&SA, 453, 969

Gredel, R., Carpentier, Y., Rouille, G., et al. 2011, A\&\&A, 530, A26

Henning, Th. \& Salama, F. 1998, Science, 282, 2204

Léger, A. \& d'Hendecourt, L. B. 1985, A\&A, 146, 81

Mattila, K., Lemke, D., Haikala, L. K., et al. 1996, A\&A 315, L353.

Mattioda, A., Cook A., Ehrenfreund P., et al. 2012, Astrobiology, Vol. 12, 841

Onaka, T. et al. 1996, Publ. Astron. Soc. Japan 48, L59.

Puget, J. L. \& Léger, A. 1989, ARAA, 27, 161.

Ruiterkamp, R., Halasinski T., Salama F., et al. 2002, A\&SA 390, 1153

Ruiterkamp, R., Cox N. L. J., Spaans M., et al. 2005, A\&A 432, 515

Salama, F. 1999, in: L. d'Hendecourt, C. Joblin and A. Jones (eds.), Solid Interstellar Matter: The ISO Revolution, (EDP Sciences, Springer-Verlag, Les Ullis), p. 65

Salama, F. 2008, in Organic Matter in Space, Proceedings IAU Symposium No. 251, S. Kwok \& S. Sandford, eds., 357.

Salama, F., Bakes, E. L. O., Allamandola, L. J., \& Tielens, A. G. G. M. 1996, ApJ, 458, 621. 
Salama, F., Galazutdinov, G. A., Krelowski, J., Allamandola, L. J., \& Musaev, F. A. 1999, ApJ, 526,265

Salama, F., Galazutdinov, G. A., Krelowski, J., et al. 2011, ApJ, 728, 154

Snow, T. P. \& McCall, B. J. 2006, ARA\&A, 44, 367

Tielens, A. G. G. M. \& Snow, T. P. (eds) 1995, The Diffuse Interstellar Bands, IAU Coll. 137, Kluwer, Dordrecht.

Tielens, A. G. G. M. 2008, ARAA, 46, 289.

van der Zwet, G. P. \& Allamandola, L. J. 1985, A\& A, 146, 76

Weisman, J. L., Lee T. J., Salama F., \& Head-Gordon M. 2003, ApJ, 587, 256 